\title{
ENZYMOSOMES: A RISING EFFECTUAL TOOL FOR TARGETED DRUG DELIVERY SYSTEM
}

\author{
SHEFRIN S., SREELAXMI C. S., VISHNU VIJAYAN, SREEJA C. NAIR* \\ Department of Pharmaceutics, Amrita School of Pharmacy, Amrita Vishwa Vidyapeetham, Amrita University, AIMS Health Science \\ Campus, Kochi, India \\ Email: sreejacnair@aims.amrita.edu
}

Received: 13 Sep 2017, Revised and Accepted: 10 Oct 2017

\begin{abstract}
The study aims to develop enzymosomes as an emerging novel drug delivery system for site-specific action. Enzymosomes utilises the specific nature of an enzyme, which is binding to a specific substrate at a controlled rate and catalysing product production step. An enzyme is encapsulated by coupling covalently to the surface of liposomes/lipid vesicles to form enzymosomes. Enzymes links through acylation, direct conjugation, physical adsorption, encapsulation methods to prepare enzymosomes with targeted action. Such novel drug delivery systems prove effective drug release and concomitantly reduces undesirable side effects of conventional treatment methods and hence showcase improvement in the long-term therapy of the disease. They are a promising substitute to conventional treatment therapies of gout, antiplatelet activities etc. Enzymosomes are newly designed supramolecular vesicular delivery systems to be useful as a tool in pharmaceutics for the raising of drug targeting and physicochemical properties and hence bioavailability. It shows beneficial effects of drugs with a narrow precision because targeting of these drugs to their site of action improves the drugs overall pharmacodynamics and pharmacokinetic profile. It also minimizes alterations in the normal enzymatic activity, thus enhancing half-life and achieve enzyme activity on targeted sites such as cancerous cells.
\end{abstract}

Keywords: Enzymosomes, Phospholipids, Superoxide dismutase, Immuno-enzymosomes, Alkaline enzymosomes, Anti-platelet enzymosomes, Targeted drug delivery

(C) 2017 The Authors. Published by Innovare Academic Sciences Pvt Ltd. This is an open access article under the CC BY license (http://creativecommons.org/licenses/by/4.0/) DOI: http://dx.doi.org/10.22159/ijap.2017v9i6.22556

\section{INTRODUCTION}

Novelty in drug delivery is an upcoming targeted system by which entities are directed to sites of action specifically [1]. Novel drug delivery system (NDDS) emerged its use in various fields like pharmaceutical, food and cosmetics industries [2]. It mainly accomplishes and overcomes the limitation of conventional drug delivery systems i.e. by transferring the entire active moiety of the drug to the site of action [3]. It also carries the drug at a rate directed manner as required by the body need through proper channels. Vesicular carriers act as the latest vehicle of choice for most drugs facing problems in bioavailability as well as extents to cells and genetic engineering, diagnosis tests, immunology techniques [4-6]. Vesicular drug delivery helps in sustaining the drug release at a predetermined rate and thus minimizing the dangerous effects of drug toxicities, providing importance in treating ocular diseases [7].

The structure of such vesicles is user-friendly and used to incorporate both hydrophilic and lipophilic drugs [8]. Most carriers are having an orderly concentric congregates of lipid bilayer where separate sites are utilized for hydrophilic and lipophilic drugs to be enclosed, finally turning to amphiphilic nature [9]. The vesicles were initially proclaimed by Bingham in 1965 who stated it as Bingham bodies. The novel carriers localize their action by spatial induction of drug bordering the diseased organ or tissue especially by chemical derivatization $[10,11]$. Researches on various drugs in glaucoma, ulcerative colitis, colon associated diseases, NSAIDS, insulin-like drugs are proven to show improved bioavailability and duration of action in humans [12]. Vesicular drug delivery decreases the cost of treatment and improves pharmacodynamics of it, especially in poorly wetting drugs [13]. It could provide great milestones in conventional chemotherapy methods where drug penetration and the permeability of cells were majorly restrained [14].

\section{Advantages of novel vesicular drug delivery system}

- It perpetuates the endurance of active drug molecule in the systemic circulation.

- Deferment the elimination reactions of promptly metabolize drugs and contributes to controlled release.
- Incorporates both hydrophilic and lipophilic drugs.

- Intensifies the stability of medicament.

- $\quad$ Cost of therapy is minimized by reducing the dose per unit formulation

- Elevate bioavailability especially in water disfavouring drugs.

- $\quad$ Selective uptake by tissues due to direct drug delivery [15].

The system utilizes a productive pathway for drug release to the site of infection thud reducing adverse effects. The vesicular drug delivery system is classified on the basis of composition as lipoidal and non-lipoidal carriers. Lipoidal carriers include sphingosomes, transferosomes, liposome, emulsomes, and virosome whereas nonlipoidal carriers include aquasomes, noisome, bilosome etc. Newer particulate carriers like lipid particles (using low and high-density lipoprotein), nanoparticles, colloidal transfer system, and polymeric micelles and various pharmaceutical carriers like cellular macromolecule are emerging as useful tools for targeted drug delivery [16].

\section{Cells to vesicles}

"Somes" are a biological cellular structure which makes up the internal organs, tissues and contains nucleic acid and genetic materials for reproduction and replication. The cell membrane incorporating the outer regions of cell in mammalian cells is made of phospholipid bilayer [17]. Such a two-layered arrangement of lipid structures makes the physiological nature mainly lipophilic.

Thus lipoidal drugs and proteins can easily cross the membranous barriers of the body due to its similar characteristics with cells lined by every organ. Such an arrangement of supramolecular lipid molecules, similar to a cell membrane is called a vesicle. Vesicles are of transport, secretory in nature which are released within our cells [18]. Exogenous vesicles can be engulfed by the cells by endocytosis and broken down into smaller fragments using lysosomal enzymes. The review mainly aims the utilization of such vesicles for targeted and site-specific drug delivery of both hydrophilic and lipophilic drugs. 
Enzymes are proteins which folds into different shapes and enable size reduced molecules to fit within them. Enzymes are biological catalysts acting on a substrate molecule which will be its target site. Enzyme converts these reactants to product molecules and thus needed by every cell to carry out a reaction at a significant rate. Since enzymes are specific and extremely selective, these mainly determine the metabolic pathway in which the cell further proceeds [19]. Enzymes are site targeted and interlocks with a lock and key mechanism to the substrate. The extent of enzyme binding to the substrate determines the response of reaction. This specificity of enzymes is exploited by combining with the concept of vesicular drug delivery.

\section{Enzymosome: an overview}

Enzymosomes is an innovative currently emerging targeted vesicular drug delivery system. Enzymosomes fundamentally uses enzymes, which are having a targeted catalytic function for a substrate, which are incorporated within cell-like structures having high lipid background. They yield newly designed liposomes, in which the enzymes are coupled covalently to the surface of lipid molecules. The liposomes so devised to provide proper micro surrounding for the enzymes to be incapacitated within them [20]. Liposomes are micro-sized vesicles consisting of a lipid bilayer enclosing with an aqueous environment. The hydrophilic drugs can be solubilised within the internal aqueous compartment and the lipophilic drugs are incorporated into the lipid bilayer membrane consisting of phospholipid-cholesterol as seen in fig. 1 [21]. The drug delivery systems of lipid-based are beneficial in special characteristics like lowering volume of distribution, interrupting drug clearance and alter the distribution of drug with enhanced capillary permeability towards the infected tissues and reducing toxicity associated with normal tissues, proving to give an efficient nanoscale drug delivering for clinical use [22].

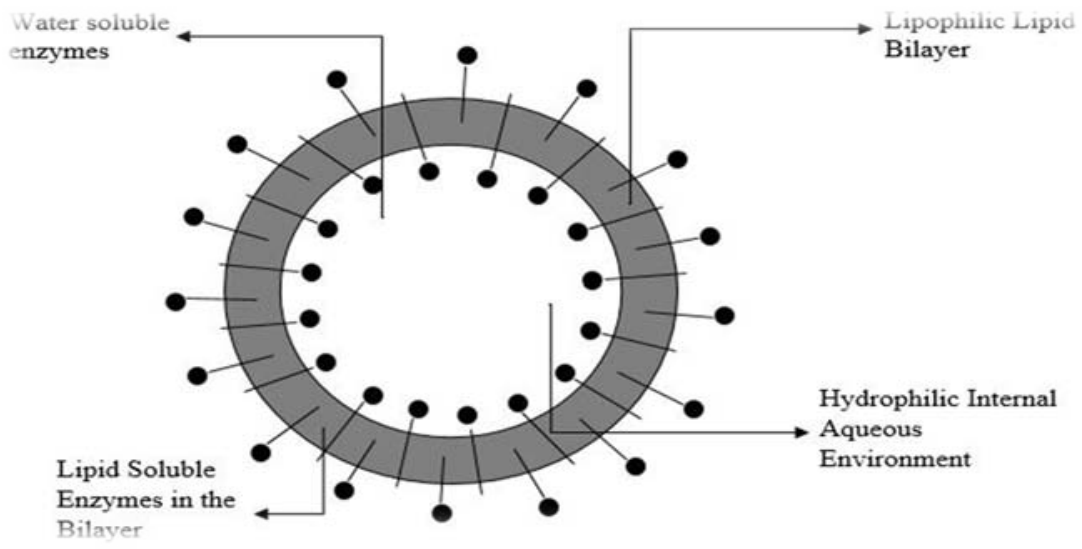

Fig. 1: Diagrammatic representation of structure of lipoid bilayer

Enzymes are having role in catalysis, site-specific pharmacological action, prodrug activation, etc. But by reviewing their low lipid membrane penetrability, if an enzyme is encased to the surface of the liposome, the degradation of the enzyme and transmutations are minimized, thus promoting their half-life along with targeted action [23]. Enzymosomes is a method of novel vesicular rate directed drug conveying system which channels the active form of the drug to the site of action and rapidly degrade them for easy uptake. Enzymes can be constrained with liposome surface by two approaches; by coupling functional hydrophobic compartments like long chain fatty acids with the enzyme or by associating the enzyme and the phospholipids of the liposome layer [24]. Using the crafted approach, enhanced therapeutic efficacy and lower side effects are visualized by carrying the therapeutic agent to the destined tissue receptors especially seen on an organ or system. Such mutated vesicles have enhanced solubility, stability, therapeutic index for the sheathed drug molecule. One of the utilization of such lipid nanocarriers is in the therapeutics of CNS disorders like epilepsy, seizures as these acts as natural attractants of BBB due to its lipophilicity [25]. The enzymes acting as therapeutic proteins are supplied through polymeric carriers like liposomes, lipoplexes, among which the attachment of enzymes to exposed areas of liposomes shows maximum response. A vesicular delivery system loaded with drugs shows precise results at the site of infection or inflammation, with least drug toxicity and side effects, useful for centrally acting drugs which have to BBB which plays a fundamental homeostatic role of the brain [26]. It also helps to embellish the bioavailability of drugs, the merest amount of drug concentration available for systemic circulation, by minimizing the purchase cost [27]. The covalently bound enzyme and liposome will have minimum alterations in the activity of the enzyme and the enzyme-loaded in a vesicle preserves its structural integrity and enzymatic activity when tested in vitro and in vivo [28]. Advances in the designing of enzymosomes are variably applicable in fields like production of new recombinant proteins, biotechnological products etc. [29, 30].

\section{Advantages of liposomes enzyme conjugates}

- Lowers the exposure to toxic drugs to sensitive tissues.

- Enhanced stability and encapsulation.

- Enhanced pharmacokinetic effects (Increase half-life, reduction in elimination).

- Couples specifically with site-specific ligands for active drug targeting.

- Increased efficacy and therapeutic index.

- Biocompatible.

- Non-immunogenic for systemic and non-systemic administered doses

- Flexible alteration in electrical properties [31].

- Non-toxic.

- Completely biodegradable.

- Site restraining effect.

\section{Disadvantages of liposome-enzyme complex}

- The production cost of liposomes are generally high since these come under the class of nanotherapeutics.

- The constituent phospholipids present in lipid vesicular structures may undergo oxidation or hydrolysis.

- Sometimes shows low solubility and shorter half-life thus reduces the bioavailability.

- The drug molecule which is encapsulated or molecules undergo fusion and leakage [32-34]. 


\section{Lipid-based drug carriers}

Lipid-based chemical systems are a monetarily accepted methodology for formulating oral, parenteral as well as topical dosage forms [35]. The safety and efficacy of such systems prove to be its trademark and is attractive for formulations, diagnostics and vaccine preparations. It is custom-fit to wide requirements by considering the cost, toxicity due to treatments like ultrasound, efficacy, and stability including indications of a disease [36]. The advantages of such systems are extensive:

- Have a low-risk portrait

- Enhanced bioavailability and less plasma concentration diversifications

- Excelling characterization and adaptability of lipophilic excipients

- Capability to locate key affairs to transfer of technology and scale-up production [37]

Vesicular systems include liposomes based vesicular carriers, lipid particulate systems and emulsions. Liposomal carriers accommodate modified liposomes, marinosome, phytosome, transferosome [38, 39]. Lipid particulate system covers lipid microparticles and lipid nanoparticle which are biocompatible in nature and devised in recent years. These are advantageous due to regulated drug release, a solid grid of stable lipids, physicochemical compatibility and preserve the active moiety from degradation. Solid-lipid nanoparticles, lipid drug conjugates and other nanostructure lipid carriers are recently developed advanced techniques of drug delivery which overtakes the polymer used nanoparticles by preventing cytotoxicity $[40,41]$. Many research works are done on the semisolid type of solid lipid nanoparticles for topical drug delivery by incorporating conventional semisolid to dispersion mixtures of the systems [42]. Emulsions encircle pickering emulsions, nanoemulsions, SLN's and self-emulsifying delivery systems [43]. Enzyme ligation with lipid carrier molecules generate enzymosomes, by preserving enzymatic activity and maintaining the structural integrity of vesicles, are the key features of enzymosomes.

\section{Applications of enzymosomes}

Enzymosomes coming underclass of lipid nanoparticulate drug delivery system, prepared primarily from phospholipids, which are arranged to a bilayer form, incorporates any substance within them, independent of solubility, electric charge, molecular weight and thus improve GIT absorption and oral bioavailability [44]. The enzymosomes can be loaded over lipid-based nanocarriers like liposomes, solid-lipid nanoparticles, inorganic nanocarriers like gold nanoparticle and magnetic nanoparticles, polymeric nanocarriers like nanogels and micelles, protein-mediated nanocarriers like super positively charged proteins etc. One assuring a current set of drugs without DNA interaction resides within ether and alkylphospholipids since cell membrane was used as the target for therapeutic intervention. In studies, these were especially effective against the clinical treatment of metastases, breast cancer, antiinflammatory action etc. [45].
Preparation of enzymosomes of surface-exposed superoxide dismutase (sod)

Binding of enzymes directly to the lipids of liposomes is a demanding work, yet they are used presently as a persuasive mechanism in the treatment of immune-mediated diseases or antibody. A model of such direct conjugated therapeutic enzyme includes SOD (Super Oxide Dismutase) [46]. The enzyme $\mathrm{Cu}, \mathrm{Zn}$ superoxide dismutase (SOD) was delineated as an innate defence mechanism which decreases the teratogenicity produced by toxic free radicals. It sets off dismutation of the toxic superoxide radical anion $\mathrm{O}_{2}{ }^{2}$ - to $\mathrm{O}_{2}$ and $\mathrm{H}_{2} \mathrm{O}_{2}$, disorganising the array of biochemical inflammatory processes induced by the free radical. SOD appears to be a promising substitute for conventional anti-inflammatory therapies, by the use of non-steroidal anti-inflammatory drugs avoiding their side effects [47]. The enzyme was not clinically acceptable due to its finite characteristics, short half-life time in the bloodstream and poor penetration into cells. Many studies was done for modifying the substrate on which the enzyme was loaded with enhanced half-life and liposomal incorporation efficiency along with clinical trials in specific risk groups like obesity, Type 2 DM, etc. [48]. To date, the most frequently used application for intracellular SOD delivery is targeting protein to cell penetrating peptides (CPPs) or protein transduction domains (PTDs). Regardless of the practical advantages of the enzyme transduction technology, the main interest of this plan is the inefficient escape from the endosome to cytosol thus leading to CPP-tagged cargoes secluded in intracellular vesicles. Proteins are of importance to biological needs and thus for controlling delivery of these peptides, modern techniques of encapsulation paved way for its adorable use in the treatment of various diseases [49].

\section{Construction of nanocarriers}

Techniques like carbon nanotubes, crosslinkednanogel matrices are used to prepare polymer colloids which encompass within nanocarriers. The productive nature of these systems depends upon the biological conditions as well as the intermolecular force of attraction. A nanocarrier must be able to carry drugs to the active site without deactivation, the drug should be released obeying the laws of kinetics, must be able to retain its stability after administration and also should actively deliver the drug with site specificity [50]. Modifications were done chemically either by covalent or non-covalent ligation. Self-assembling of proteins and nanocarriers were another alternative.

Through physical adsorption method, spontaneous binding is achieved by utilizing the driving electrostatic force as seen in fig. 2 [51]. Nanoparticles can be congregated from a variety of materials to covetable geometries and configurations and thus to acquire useful functionalities and properties [52]. Conventional chemotherapy treatments bear various adverse reactions along with the inability to access the core of the disease. Thus nano-sized polymeric carriers prove to be selectively and specifically cell-targeted drug delivery $[53,[54]$.

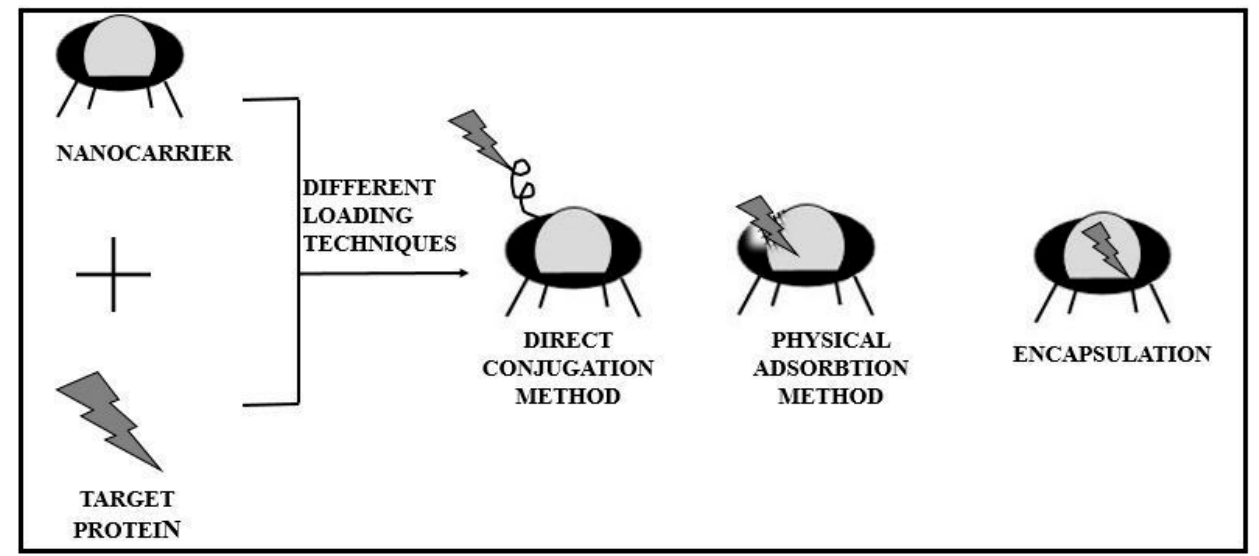

Fig. 2: Schematic of three loading methods for preparing protein/nanocarrier composites 
The launching of SOD over the liposomal surface is conventional to be more beneficial than an encapsulation of enzyme in liposomes, since the release of the enzyme from the liposomes at the site of inflammation may not be needed to achieve therapeutic activity. To accomplish surface localization of SOD on the enzymosomes, the method of acylation by covalent linkage of palmitic acid to $\mathrm{q}-\mathrm{NH}_{2}$ groups of SOD (Ac-SOD) [55]. The studies speculated that the procedure will result in a more hydrophobic enzyme with enhanced affinity for the liposomal bilayers. Also by exposing SOD onto the external liposomal surface, we also intended to target SOD to inflammatory sites. For this liposome were prepared with a long circulation time for obtaining localization at the inflammation sites and acts as efficient intracellular drug delivery method [56] Through these studies, it was convincingly visualized that longcirculating liposomes (LCL) tends to localize preferentially at inflammation sites after i. v. administration. This preferential localization could be due to the inflammatory response provoking a locally maximized capillary permeability allowing liposome extravasation. Therefore, LCL has attractive prospects for the targeted delivery of Ac-SOD. SOD and Ac-SOD was incorporated in two types of liposomes: PEG-coated LCL (PEG liposomes) and non-PEG-liposomes containing stearyl amine (SA-liposomes). The enzymes are sensitive to physical and chemical stress like thermal treatment, thus are protected within lipid bilayers maintaining their native conformation [57]. Both liposomal formulations were characterized with regard to incorporation efficiency, zeta potential, retention of enzymatic activity and externally exposed enzyme activity.

The studies done for preparing and optimizing SOD enzymosomes was mainly to enhance longer time of circulation of human blood and accumulate the enzyme at the target site, therefore maintaining the enzyme flawless within the lipid structure [58]. Hydrophilic range therapeutic enzymes are retained or encapsulated within the inner aqueous space of prepared vesicles. A liposome, which exhibits catalytic action in its intact form, i.e. before it gets destroyed. The enzymes could be bound to liposomes by two approaches:-

- By interacting the hydrophobic anchoring molecules like the long chain fatty acids to the enzyme.

- By initially linking enzyme directly to the liposome bilayer components of the phospholipids.

As a result of the first process, the enzyme is assimilated to the liposomal membrane, and in the latter step, the docking is between liposome bilayer and coupling reaction take place with the liposomal surface. Both the processes are having difference in,

- The stability of the enzyme-liposome complex.

- A number of enzyme molecules which are entrapped/displayed to the outer lipid bilayer.

- The nature of modified enzymosomes, since there are many molecules to which the enzyme could be docked, example phospholipids, long chain fatty acids or polymer-like substances attached to phospholipids.

- The method of preparation is generally chosen according to the diverse cases of therapeutic needs where enzyme delivery mediated by enzymosomes is required.

A schematic diagram depicted in Gaspar MM et al., where the ligation of enzymes to the internal hydrophilic environment, i.e. native enzyme occurs and to the lipid bilayer membrane of liposomes by the Ac-enzyme [59]. When the liposomes are intact, the enzyme in the interiors are not available for reaction. But when the enzymes are directly attached to the lipid membrane, the enzyme is available for catalytic action even before the liposomal destruction.

During the unification of hydrophilic enzyme to the acyl residual chains of lipids, the process of acylation (addition of acyl functional group) occurs resulting in the formation of Ac-enzyme. This result in diversion of enzyme from hydrophilic to hydrophobic microenvironment. The attained hydrophobicity level is inflected by the length and number of fatty chains joined to the surface of the enzyme. The conservancy of other equities of the modified enzyme must be evaluated by using the basic strategies of conjugation. An example, L-Asparginase enzyme used for treatment of acute lymphoblastic leukaemia, withholds $100 \%$ of its catalytic action, if the active site of enzyme is hindered with the substrate while conjugation. For augmenting a fine dose of Ac-enzyme to the liposomal structure and for enabling proper release of enzymes, different strategies are used. The partly insertion of Ac-enzyme into lipid bilayer or concealed within the hydrophobic lipid vesicular matrix. The association Ac-enzyme to the lipid bilayer membrane is mainly dependent on several factors, including electrostatic interactions between the charges associated with enzymes [60]. The efficiency of the Ac-enzyme incorporated to liposome is determined by calculating the ratio between catalytic values estimated during the intact life period versus the disrupted enzymosomes. The ideal characteristics of liposomes play a very useful role during construction of enzymosomes which include, the size of the vesicle, composition, ionic charge, etc.

\section{Acylated-sod liposomes}

Generally, the SOD enzymosomes was a homogenous film obtained by dispersing aqueous solution containing SOD and thus the nonbounded enzyme was extruded by ultracentrifugation separation method. The prepared enzymosomes were characterized by the research work of Gaspar MM et al, by different parameters which ensured that the enzymosomes is standard in its characteristics. The different parameters included where;

- Liposome mean particle size (diameter) was analysed using dynamic light scattering.

- Protein coupling to liposomes was assessed after disruption of the liposomes with Triton X-100 and sodium dodecyl sulphate (SDS).

- Free amino acid groups and lipids were determined.

- Phospholipids by colourimetric assay.

- The enzymatic activity of Ac-SOD and SOD formulations was determined.

- The enzymatic ability to decrease the speed of autoxidation of epinephrine to adrenochrome was assessed.

- The total activity of enzyme within SOD or Ac-SOD enzymosome was determined by initially preparing a series of dilutions to obtain a final concentration of protein.

- The enzymatic activity of the enzyme exposed to the external surface was measured.

- Zeta potential was analysed.

- The electric field intensity and scattering angle were found.

- The thermotropic behaviour of the membrane phospholipids of enzymosomes was found [61].

The results of various works in chemical alterations of SOD were presented innovative methodologies for characterizing Ac-SOD and analysed if it was superior over plain SOD and other pharmaceutical preparations.

- The surpassing parameters for Ac-SOD evidenced higher affinity of it to the hydrophobic area of liposomal bilayer when compared to free SOD.

- The incorporation efficiency of Ac-SOD in SA containing enzymosome was set side by side to PEG-liposome, where having lower initial (protein/lipoprotein) ratio, because of competition between Ac-SOD with cholesterol for inclusion between phospholipids.

- The varied electrostatic interactions suggested that positively charged SA-liposome advantageous were as due PEG at the lipid surface, it reduced lipid-protein charged interactions.

Thus Ac-SOD presented substantial activity for the incorporated enzyme, which acts independently depending on, rate and extend of enzyme release, thus producing a different mechanism of action. Thus the undertaken work confirms that the designed enzymosome 
is significant potential since even in the presence of surface PEG chains, the PEG-enzymosome converted the substrate. Thus, it was not a barrier and the release of the enzyme was not a prerequisite for producing dismutation action at the inflamed site. If the Ac-SOD enzymosome could be prepared with circular micro-reservoirs, it could be markedly ample for expressing its action without disruption and also for a sustained release pattern of the enzyme. Similar modifications are done for peptides and polymers which makes an important drug targeting pathway [62]. Therefore the Acenzymosome could be a replaceable therapeutic agent for an intense effect in rheumatoid arthritis and longer flowing active particles for reperfusion pathologies. The affinity of SOD for negatively charged lipid molecules accounts for at least part of its ability to protect lipid membranes from oxygen-induced injury and are valuable tools for studying membrane structure and dynamics [63].

\section{Designing of immuno-enzymosomes having enhanced enzyme} targeting capability and cell binding properties

The potency of traditional anticancer drugs for the treatment of chemotherapy is confined due to the inadequacy of discrimination between normal as well as cancerous cells. On reviewing the studies on immuno-enzymosomes could be used to target enzymes to for achieving site peculiar activation of anticancer prodrugs. The potency of generating a sole well portrayed liposomal system which is having a binding capacity to variety targeted ligands is significant worth. These methods offer the hiding of enzyme within small packet like structures and releasing them on reaching action site [64]. It was stated that enzyme, $\beta$-glucuronidase, which was proficient in stimulating anthracycline glucuronide prodrugs, when coupled with immunoliposomes surface aimed against ovarian cancer cells (OVCAR-3). Thus, by cleansing commercially accessible enzyme $\beta$-glucuronidase (GUS) an immune-enzymosome formulation could be prepared which had a 2 times increase in its enzyme specific activity when incubated with ovarian cancer cells
[65]. The principle involves fusion of a cell unique antibody with (liposome) immunoliposome enclosing the chemotherapeutic agent, thus providing a probability for selective drug transmission and cellspecific cytotoxicity as seen in fig. 3 [66]. This results in new derivatives of therapeutic proteins having minimum immunegenicity. The antibody-enzyme complex was administered, pursued by injection of comparable non-toxic prodrug after binding of the complex with cancerous cells and cleared from the blood and tissues. Targeted by the enzyme's action, the prodrug is converted to active cytotoxic molecule within the concurrence of tumour cells, therefore leading to its selective wiping out.

\section{Advantages}

- More than one enzyme moiety could be admitted in one targeted carrier system.

- Enhanced enzyme density at the cancer cell surface, thus providing efficient conversion of the prodrug.

- The enzyme GUS was preferred due to its superiority over other enzymes, which were present localized intracellular.

- They cause limited activation of hydrophilic glucoronide drugs since they have only low penetration. Thus lowering immunogenicity problem.

The GUS enzyme due to its bulky steric hindrance sometimes could negatively affect the coupling with cells. But studies showed that even increasing the enzymatic density over the surface provided large enzymatic targeting. In studies of Szczupak et al., the electro some was used as a tool for the release and action of a cascade of enzymes overcoming the drawback of the low number of enzymes on the surface [67]. The GUS obtained was initially purified and Fab' fragments were developed. Using these immuno-liposome or the enzymosome was generated followed by its characterization.

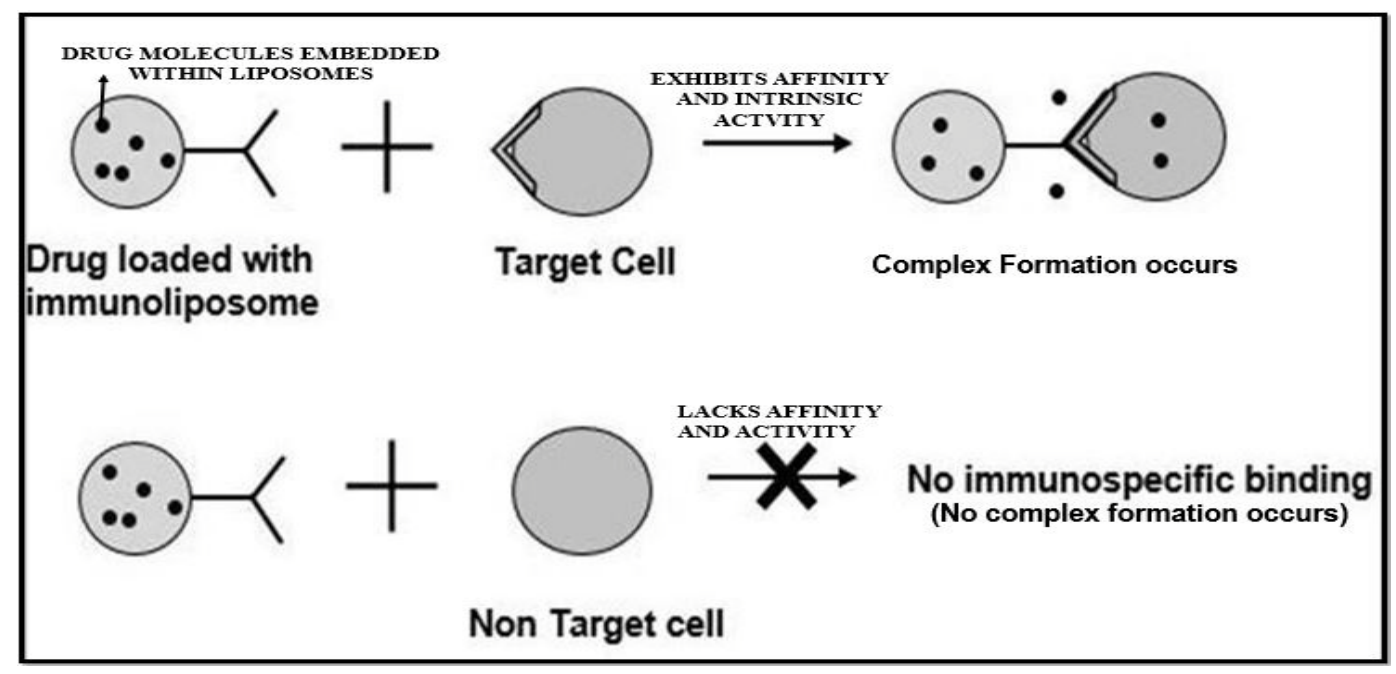

Fig. 3: Specificity of immuno-enzymosomes to target cells

The review of the research work, for the most part, was done to cite certain important milestones in the field of nanoscience, which can be used as leads for developing efficient drug delivering methods for uncompromising diseases. The main objectives of the study were to preserve maximum enzyme density at cell environment and to quantitatively enhance the enzyme without its aggregation by undergoing additional conformational changes, which determines the enzymes efficacy and selectivity [68].

As the experiment progresses, the purification of commercially available GUS increased two folds its enzymatic action. Thus terminating, the density of enzyme up to the level of a steric hindrance for interacting with a target antigen could be an effective strategy for producing immuno-enzymosomes with maximum enzyme targeting capability as seen in fig. 4 [69]. The major application of the immuno enzymosomes was in the diagnosis and treatment of ovarian carcinomas. Even during the initial stages itself, the cancer cells could spread up to the peritoneal cavity.

The usual surgical removal of tumorous mass followed by chemotherapy is done regularly. Thus, for a complete curing with the full elimination of residual matters which could otherwise further damage the normal cells. The studies thus confirm that by giving the immuno-enzymosomes preparation i. p for antibodydirected enzyme prodrug therapy, the peritoneally injected drug could easily gain access to targeted cells to which the enzyme has to bind. 


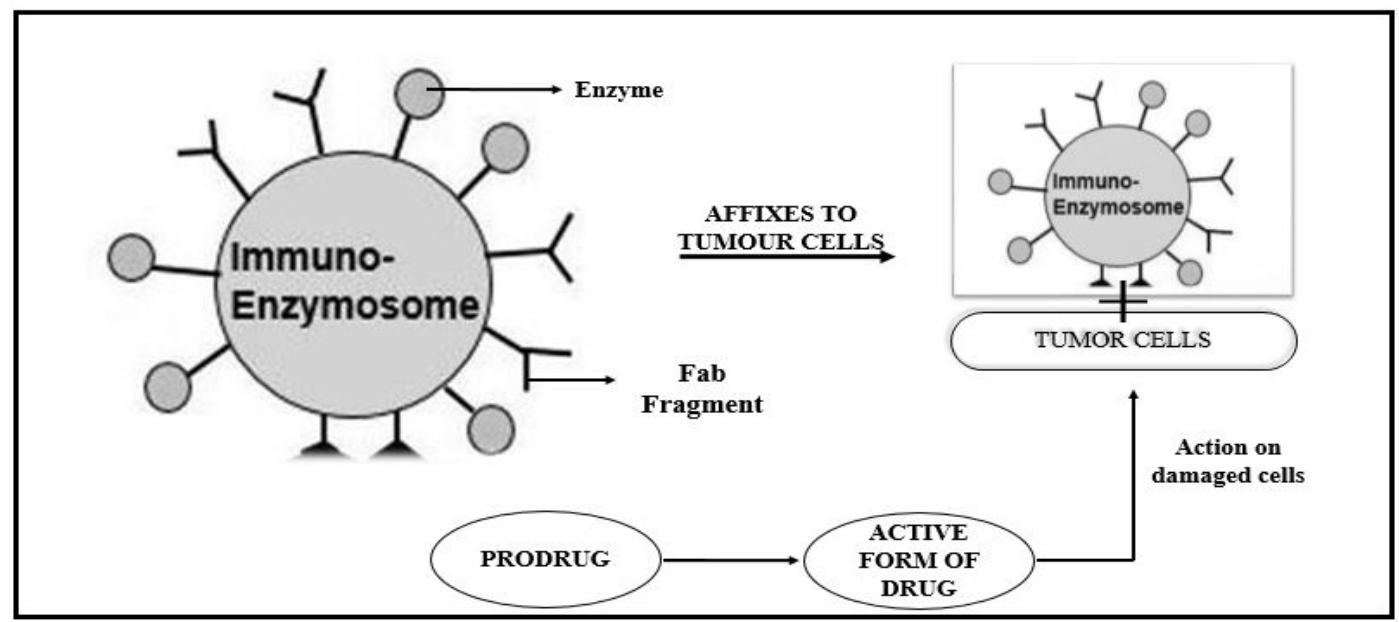

Fig. 4: Enzyme bound drug targeting tumour cells

\section{Production alkaline enzymosomes loaded with Bacillus fastidious}

The study done by Tan $\mathrm{Q}$ et al. was to judge the promising action of a novel alkaline enzymosome for delivering the enzyme, Uricase from Bacillus fastidious (UBF) and embellish its biochemical activity, therapeutic use and pharmacological characteristics [70]. Uricase have a normal physiological action to lower the plasma uric acid level by undergoing catalysis of uric acid by oxidation reaction [71]. It was proven from studies that it took less time for UBF loaded in the novel alkaline enzymosomes (ESUBFs) to diminish the plasma uric acid concentration to the normal level from higher, compared to the time taken by free UBF [72, 73]. Their results showed that ESUBFs effortlessly delivers UBF and positively increasing the total area under curve (AUC), half-life $\left(\mathrm{t}^{1 / 2}\right)$ and catalytic activity as well as modifying its biochemical and pharmacological characteristics [74]. Thus ESUBFs could be a favourable second choice solution to problems like gout and hyperuricemia [75]. It was the additionally accountable advantage of the expanded in vivo uricolytic action could be of great clinical implication since the clinical dosage given as well as the side effects produced during the normal dosage of UBF formulation could be much considerably reduced via conjugation [76]. In the work, the development of novel alkaline UBF enzymosomes was profitably developed to indeed improve the pharmaceutical, biological and biochemical properties and exploited for hyperuricemia effects of enzyme uricase from Bacillus fastidious. In research work of Szczurek et al. the oral delivery of uricase enzyme was found to reduce uric acid levels in hyperurecemia rat model [77, 78]. Uricase enzyme had constitutional advantages like enhanced selectivity with efficient, fast reactivity along with disadvantages like short in vivo half-life with limited therapeutic usage. The main aim of encapsulating an enzyme within an enzymosomes is to regulate its delivery and also to sustain its molecular structure intact.

The research work included the catalytic activity of the UBF loaded in the novel alkaline enzymosomes (ESUBFs) in vitro, which suggested almost 3 folds that of free UBF at the optimum pH. Following intravenous (i. v.) administration in study model, ESUBFs provided a significantly higher area under the plasma concentration (AUC) and longer circulation half-life $(\mathrm{t} 1 / 2)$ compared with free UBF, similar to studies by Xiong $\mathrm{H}$ et al. [79]. The preparatory security assessment actively exhibited that ESUBFs was acceptable when injected through the parenteral route of administration thus used in emergencies of enzyme replacement for curing enzyme deficit diseases [80]. The systematic investigation trends indicate well the maximized activity of encapsulated biochemical and pharmacological characters of alkaline enzymosomes. Therefore enzymosomes could be a preferred alternative for the formulation of UBF and ESUBFs which might be a preferred alternative to therapeutically cure hyperuricemia and gout.

\section{Antiplatelet activity of CD 39 enzymosomes}

The endothelial cells of body consist of an enzyme CD39/NTPDase-1 which is expressed on the opening side of the cell. It physiologically has an ability to rapidly metabolize ATP and ADP, along with AMP and minimizing the platelet sensitivity to the major agonists. Thus, motivation existed to examine therapeutic anti-platelet 'enzymosomes' formulations that consist of CD39 integrated within liposome lipid bilayers. Initially, CD39 enzymatic activity was optimized which appears to be dependent on the expression of either of its transmembrane domains [81]. The full-length human CD39 was generated by using a yeast expression system as a model which was purified and reconstituted within a suitable lipid vesicle chosen accordingly. The dephosphorylation and formation of ADP and ATP were taken as parameters for finding it's catalytic efficiency and was determined both for detergent solubilized CD39 and when reconstituted within a lipid membrane. The CD39 containing lipid vesicles ability to inhibit platelet activation induced by ADP, collagen and thrombin was defined in vitro by platelet aggregometry method. A murine model of thromboplastin induced thromboembolism was utilized to find out the effectiveness and therapeutic applicability of intravenously administered CD39 enzymosomes in limiting platelet consumption and death. The reconstitution of human CD39 in lipid vesicles caused a reduction of the $\mathrm{Km}$ value of nearly an order with aconcomitant enhancement in both ADPase and ATPase catalytic efficiency [82]. The platelet activation by ADP, collagen, or thrombin was effectively inhibited after administration of CD39 lipid vesicles thus effectively inhibited platelet aggregation when platelets were activated and produced a platelet disaggregation response.

One drawback obtained from studies was that treatment with CD39 lipid vesicles enormously limited the reduction in platelet counts caused by thromboplastin. The incorporation of the enzyme into a lipid bilayer significantly enhanced CD39 enzyme activity when compared to its solubilized counterpart. Thus, studies revealed treatment with CD39 enzymosomes minimized platelet consumption and death rate in the animal model. Thus prepared CD39 enzymosomes could be a useful therapeutic option for complementing other existing anti-platelet therapies characterized by platelet thrombus formation [83].

\section{Generation of streptavidin-liposomal conjugates for targeted} ligand specific applications

The tetrameric biotin-binding protein, Streptavidin confined from Streptomyces avidinii is having a low degree of nonspecific binding in immunohistochemistry applications. Thus, it is a quintessential choice in various detection systems [84]. It belongs to the avidin group of proteins which functions as antibiotics and its specific interaction with biotin molecules makes it useful in nonradioactive detection systems. Biotin is a vitamin in living cells demanding in different biological processes, including cell growth [85]. Formerly, when biotin is attached to a molecule, the biotin tag is used to assist the progress of affinity purification of molecules using immobilized biotin-binding protein. Thus the avidin-biotin interaction is applied in Enzyme-linked immunosorbent assay (ELISA), Immunohistochemistry (IHC), Cell-surface labelling, Fluorescence-activated 
cell sorting (FACS) etc. [86]. Thus, under optimum conditions Streptavidin attached to liposome result in well-characterized protein-liposome conjugate. The resulting targeted vesicle system retains more activity with respect to their size and binds more firmly to the biotinylated targeting ligands. The coupling of natural circulating cells with enzymes for drug delivery has gained interest in later years [87].

In the studies, the streptavidin is noncovalently coupled to biotin which is a vitamin and phosphatidylethanolamine which is a class of phospholipid. The phospholipid was chosen due to its primary role in distributing out negative charge produced by anionic membrane phospholipids [88]. The researchers used the extrusion method to prepare the sample by dissolving the lipid mixture in a solvent which was coated over a tube a dried to a thin film by passing a stream of appropriate gas under high vacuum [89]. The biotinylation process involved the covalent coupling of a protein or a nucleic acid with biotin. Biotin combination with streptavidin, a member of avidin family is with high affinity and fast action and thus in many fields of biotechnology to separate such biotinylated compounds of interest. The liposome vesicles were incubated with streptavidin for accomplishing its binding and at a constant ratio of streptavidin to lipid molecules when maintained, maximum coupling efficiency was obtained [90]. This efficient communication between streptavidin and biotin containing lipid molecules is utilized in the indirect targeting procedures. Other drug discoveries like photoaffinity is used as additional mechanisms for molecular interactions and targets for the binding site via activation through light [91].

Another method investigated in studies was the covalent binding streptavidin to two lipid derivatives, which contained thiol groups to initiate the reaction [92]. In the research work done in the reference article, the maleimide derivatives of lipids were cross-connected better due to the presence of long spacing reactive arms [93]. The enzyme is then coupled with these liposomes and is designed in such a manner to use its ability to conjugate in a specific manner with membrane-linked antigens, producing different nanocarriers with physicochemical features efficient as a drug delivery system [94]. The biotinated antibody was coupled with enzyme-linked with liposomes which had wide in vivo and in vitro applications. Such applications are useful in retention of hydrophilic drugs and fluorescent groups in water-loving compartments of targeted liposomes for cell surface activity [95]. The conjugates of small size preparations proves longer half-life and thus retains their capacity and action more within the plasma,varying the infection and acute reaction severity [96].

\section{CONCLUSION}

Enzymosomes are suitably engineered lipid constructions in which there is a suitable mini-environment for the enzyme to be immobilized within lipid structures or covalently coupled to the outer side of lipids. It has its major application in easiness in permeation as well as for targeted drug arrival at tumorous cellular sites [97]. These vesicular systems have wide flexibility for drug development to overcome several side effects and bioavailability problems. The approach of nanosystems of delivering drugs to the CNS by permeating through BBB is promising lead for enhancing treatment methods like in different type of malignancies [98]. Various prodrugs, as well as charged proteins along with complexes, are delivered to the brain by the method thus increasing the concentration of drug linearly. Even though different carrier nucleus has disadvantages like oxidative stress damage, in spite of all they have a major role in selecting and targeting and brings a newer origin to conventional drug therapies [99]. Various methods like biotinylation, pegylation are the latest innovative trends for targeted drug delivery. There are various lipoidal and nonlipoidal vesicular carrier nanoparticles utilized for sustained drug release and cellular targeting. Enzymosomes as one of the lipid bio carrier along with others like ethosome, transferosome, pharmacosome, virosome, and non-lipoidal ones like niosomes, aquasomesetc. [100]. Each carrier is now proven to be successful in their action though various studies. From studies of CA Valeetal., it was proven that PEG-SOD enzymosomes were having an adequate action to be used therapeutically. Liposomes are now becoming clinically accepted since they maintain the level of toxicity to a minimum and attains enhanced efficacy when compared with free supplements. Review of
Nair et al., shows that emulsomes are proven carriers for loading low water-soluble drugs and thus variable bioavailability when compared free drug. Thus enzymosomes can be also an assuring carrier nucleus to achieve a newer generation of flexible drug designing system in future.

To advance the better applications of carrier-mediated protein delivery the different strategically approved methods. The therapeutic enzymes are integrated to carriers of the polymeric nature of the hydrophilic area of lipid vesicles and the hydrophobized forms assimilated to lipid bilayers of vesicles. Not one thing above specified can retain the activity of therapeutic protein fully. Other than therapeutic enzymes another method of plan is that, its coupling to the outer surface of liposomes, by which scientific know-how was developed for an antibody with drug preparation. Complexation of the enzyme with lipid nature carriers brought about enzymosomes. The seductive characteristics of enzymosomes is its enzymatic activity preservation and restraining structural integrity. The conception of consolidating the drug to lipid vesicles for a more exceptional targeting of the drug at the apt tissue terminal is widely accepted. It is discernible that different deformable and lipid, rigid supramolecular vesicular constructs have tremendous potential to deliver drugs for targeted delivery of different bioactive. During last years, repeated researches have been going on to achieve the target with the goal of enhanced targeting and limiting the dosing frequency. To acquire these efforts, these can be formulated in a semi-liquid or liquid drug delivery systems with corresponding matching lipid composition. The novel vesicular systems actively revealed their therapeutic potential to genetic levels from topical one. Using the notions of supramolecular chemistry, an enzymosome, a novel vesicular system is capable of acquiring the mentioned traits.

\section{ACKNOWLEDGEMENT}

We are immensely obliged and thankful to Dr. Sabitha M., Principal, Amrita School of Pharmacy, Amrita Vishwa Vidyapeetham, Amrita University. We also extend our gratitude to The Department of Pharmaceutics for catering legitimate facilities for carrying out the research work.

\section{CONFLICT OF INTERESTS}

\section{All authors have none to declare.}

\section{REFERENCES}

1. Kumar R, Kumar S, Jha SS, Jha AK. Vesicular system-carrier for drug delivery. Chem Sinica 2011;2:192-202.

2. Kunwarpuriya AS, Doke VV, Changedia S, Khutle NM. Sphingosome: a novel vesicular drug delivery system. Eur J Pharm Med Res 2015;2:509-25.

3. Lade S, Burle S, Kosalge S, Kannao S. Lipid-based drug delivery systems: a comprehensive review. Int J Innov Pharm Sci Res 2014;2:2465-75.

4. Fathima KM, Nitheesh A, Paul A, Nair SC. Sphingosome vesicular system. Int J Pharm Sci Rev Res 2016;39:208-13.

5. Zishan M, Kushwaha P, Singh K. An overview of: vesicular drug delivery system. World J Pharm Pharm Sci 2017;6:546-60.

6. Doke V, Kelan D, Khadse D, Khutle N, Chaudhari Y. Ethosome: a novel vesicular drug delivery. World J Pharm Res 2015;4:1199210.

7. Biswas GR, Majee SB. Niosomes in ocular drug delivery. Eur J Pharm Med Res 2017;4:813-9.

8. Vyas SP, Khar RK. Targeted and controlled drug delivery. 1st ed. New Delhi: CBS Publisher; 2002.

9. Akiladevi D, Basak S. Ethosomes-a noninvasive approach for transdermal drug delivery. Int J Curr Pharma Res 2010;2:1-4.

10. Jain S, Jain P. Transferosomes: a novel vesicular carrier for enhanced transdermal delivery: development, characterization and performance evaluation. Drug Dev Ind Pharm 2003;29:1013-26.

11. Nandure HP, Puranik P, Giram P, Lone V. Ethosome: a novel drug carrier. Int J Pharm Res Allied Sci 2013;2:18-30.

12. Sreelatha D, Brahma CK. Colon targeted drug delivery-a review of primary and novel approaches. J Global Trends Pharm Sci 2013;4:1174-83. 
13. Touitou E, Godin B, Dayan N, Weiss C. Intracellular delivery mediated by an ethosomal carrier. Biomaterials 2001;22:3053-9.

14. Bhingare U, Khadabadi SS, Shinde N. Pharmacosomes: a novel drug delivery system. Int J Pharm Res Allied Sci 2014;1:14-20.

15. Moghimipour E, Handali S. Liposomes as drug delivery systems: properties and applications. Res J Pharm Biol Chem Sci 2013;4:169-85.

16. Pawar P, Kalamkar R, Jain A, Amberkar S. Ethosomes: a novel tool for herbal drug delivery. Int J Pharm Pharm Sci 2015;3:192-202.

17. Overholtzer M, Brugge JS. The cell biology of cell-in-cell structures. Nat Rev Mol Cell Biol 2008;9:796-809.

18. Engel H, Rondeau E, Windhab EJ, Walde P. External surface area determination of lipid vesicles using trinitrobenzenesulfonate and ultraviolet/visible spectrophotometry. Anal Biochem 2013;442:262-71.

19. Villalonga ML, Diez P, Sanchez A, Gamella M, Pingarrón JM Villalonga R. Neoglycoenzymes. Chem Rev 2014;114:4868-917.

20. Akbarzadeh A, Sadabady RR, Davaran S. Liposome: classification, preparation and applications. Nanoscale Res Lett 2013;8:102-11.

21. Sharma S, Mishra L, Grover I, Gupta A Kaur. Liposome: vesicular system an overview. Int J Pharm Pharm Sci 2010;2:11-7.

22. Zylberberg C, Matosevic S. Pharmaceutical liposomal drug delivery: a review of new delivery systems and look at the regulatory landscape. Drug Delivery 2016;23:3319-29.

23. Manish G, Vimukta S. Targeted drug delivery system: a review. Res J Chem Sci 2011;1:135-8.

24. Kamal K, Garg G, Harikumar SL, Aggarwal G. Potential role of nanotechnology for skin drug delivery. World J Pharm Pharm Sci 2016;5:428-53.

25. Pardridge WM. Drug transport across the blood-brain barrier. ] Cereb Blood Flow Metab 2012;32:1959-72.

26. Marianecci C, Rinaldi F, Hanieh PN. Drug delivery in overcoming the blood-brain barrier: role of nasal mucosal grafting. Drug Des Dev Ther 2017;11:325-35.

27. Salunkhe SS, Bhatia NM, Kawade VS, Bhatia MS. Development of lipid-based nanoparticulate drug delivery systems and drug carrier complexes for delivery to brain. J Appl Pharm Sci 2015;5:110-29.

28. Cui S, Zhi D, Zhao Y. Cationic liposomes with folic acid as targeting ligand for gene delivery. Bioorg Med Chem Lett 2016;26:4025-9.

29. Gorle S, Sewbalas A, Ariatti M, Singh M. Ligand-tagged cationic liposome facilitates efficient gene delivery to folate receptors. Curr Sci 2016;3:662-70.

30. Pattni BS, Chupin VV, Torchilin VP. New developments in liposomal drug delivery. Chem Rev 2015;115:10938-66.

31. Andhale VA, Patil PR, Dhas AU, Chauhan PD, Desai SV. Liposome: an emerging tool in drug carrier system. Int J Pharm Technol 2016;8:10982-1011.

32. Popovska O, SimonovskaJ, Kavrakovski Z, Rafailovska V. An overview: methods for preparation and characterization of liposomes as drug delivery systems. Int J Pharmphytopharm Res 2014;3:182-9.

33. Saroj S, Baby DA, Sabitha M. Current trends in lipid-based delivery systems and its applications in drug delivery. Asian J Pharm Clin Res 2012;5:4-9.

34. Kavitha AN, Deepthi V. Liposomal drug delivery system-a review. RGUHS J Pharm Sci 2014;4:47-56.

35. Amidon GL, Shah VP. A theoretical basis for a biopharmaceutical drug classification: the correlation of in vitro drug product dissolution and in vivo bioavailability. AAPS J 2014;16:894-98.

36. Leelarungrayub J, Manorsoi J, Manorsoi A. Anti-inflammatory activity of niosomes entrapped wit Plai oil (ZingibercassumunarRoxb.) by therapeutic ultrasound in rat model. Int J Nanomed 2017;12:2469-76.

37. Shrestha H, Bala R, Arora S. Lipid-based drug delivery systems. J Pharm 2014;10:1-10.

38. Devarajan V, Ravichandran V. Nanoemulsions: as modified drug delivery tool. Pharmacie Globale 2011:4:1-6.

39. Kumar R, Kumar S, Jha SS, Jha AK. Vesicular system-carrier for drug delivery. Pharm Sinica 2011;2:192-202.
40. Soni K, Kukereja BK, Kapur M, Kohli K. Lipid nanoparticles: future of oral drug delivery and their current trends and regulatory issues. Int J Curr Pharm Rev Res 2015;7:1-18.

41. Kakadia PG, Conway BR. Solid-lipid nanoparticles: a potential approach for dermal drug delivery. Am J Pharmacol Sci 2014;2:1-7.

42. Trombino S, Mellace S, Cassano R. Solid lipid nanoparticles for antifungal drugs delivery for topical applications. Ther Delivery 2016;7:639-47.

43. Badilli U, Turk CTS, Amasya G, Tarimci N. Novel drug delivery system for dermal uptake of etofenamate: semisolid SLN dispersion. Curr Drug Delivery 2017;14:386-93.

44. Das S, Chaudhury A. Recent advances in lipid nanoparticle formulations with the solid matrix for oral drug delivery. AAPS PharmSciTech 2011;12:62-76.

45. Rajitha P, Gopinath D, Biswas R, Sabitha M, Jayakumar R. Chitosan nanoparticles in drug therapy and infectious diseases. Expert Opin Drug Delivery 2016;13:1177-94.

46. Gaspar MM, Martins MB, Corvo ML, Cruz MEM. Design and characterization of enzymosomes with surface-exposed superoxide dismutase. Biochim Biophys Acta 2003;16:211-7.

47. Corvo ML, Marinho HS, Marcelino P. Superoxide dismutase enzymosomes: carrier capacity optimization, in vivo behaviour and therapeutic activity. Pharm Res 2015;32:91-102.

48. Sabitha K, Venugopal B, Rafi M, Ramana KV. Role of antioxidant enzymes in glucose and lipid metabolism in association with obesity and type 2 diabetes. Am J Med Sci 2014;2:21-4.

49. Kobsa S, Saltzman WM. Bioengineering approaches to controlled protein delivery. Pediatr Res 2008;63:513-9.

50. Anwekar H, Patel S, Singhai AK. Liposome-as drug carrier. Int J Life Sci Pharma Res 2011;2:945-51.

51. Solaro R, Chiellini F, Battisti A. Targeted delivery of protein drugs by nanocarriers. Materials 2010;3:1928-80.

52. Peer D, Karp JM, Hong S, Farokhzad OC, Margalit R, Langer R Nanocarriers as an emerging platform for cancer therapy. Nat Nanotechnol 2007;2:751-60.

53. Sivasankar M, Katyayani T. Liposomes: the future of formulations. Int J Res Pharm Chem 2011;1:259-67.

54. Zhao H, Lin ZY, Yildirimer L, Dhinakar A, Zhao X, Wu J. Polymerbased nanoparticles for protein delivery: design, strategies, and applications. J Med Chem 2016;4:4060-71.

55. Liu G, Li K, Wang H. Polymeric micelles based on PEGulated chitosan-g lipoic acid carrier for efficient intracellular drug delivery. J Biomater Appl 2016;31:1039-48.

56. Fanum M. Colloids in drug delivery. $1^{\text {st }}$ ed. USA: CRC Press; 2016.

57. Venkatesh DN, Rao P. Nanoparticles for cancer treatment-a comprehensive review. World J Pharm Pharm Sci 2016;5:481-99.

58. Gaspar MM, Boerman OC, Laverman P. Enzymosomes with surface-exposed superoxide dismutase: in vivo behaviour and therapeutic activity in model of adjuvant arthritis. J Controlled Release 2007;117:186-95.

59. Xu G, McLeod HL. Strategies for enzyme/prodrug cancer therapy. Clin Cancer Res 2001;7:3314-24.

60. Bisswanger H. Enzyme assays. Perspect Sci 2014;1:51-5

61. Chen T, Wang R, Lu T, Liang G, Lu T. Modification with liposomes by the dansyl-labeled heterobifunctional crosslinker. J Biomater Appl 2011;26:117-25.

62. Yang Y, Wang J, Shigematsu H. Self assembly of size-controlled liposomes on DNA nanoplates. Nat Chem 2016;8:476-83.

63. Shekhar C. Lean and mean: nanoparticle-based delivery improves the performance of cancer drugs. Chem Biol 2009;16:349-50.

64. Saeed HM, Abdel-Fattah YR, Gohar YM, Elbaz MA. Purification and characterization of extracellular Pseudomonas aeruginosaurate oxidase enzyme. Pol J Microbiol 2004;53:45-52.

65. Wu J, Lu S, Zheng Z, Zhu L, Zhan X. Modification with the polysialic acid-PEG copolymer as a new method for improving the therapeutic efficacy of proteins. Prep Biochem Biotechnol 2016;46:788-97.

66. Szczupak A, Aizik D, Morais S. The electrosome: a surfacedisplayed enzymatic cascade in a biofuel cell's anode and a high-density surface-displayed biocathodic enzyme. Nanomaterials 2017;7:1-17. 
67. Secundo F. Conformational changes of enzymes upon immobilisation. Chem Soc Rev 2013;42:6250-61.

68. Tan Q, Zhang J, Wang N. Uricase from Bacillus fastidious loaded in alkaline enzymosomes: enhanced biochemical and pharmacological characteristics in hypouricemic rats. Eur J Pharm Biopharm 2012;82:43-8.

69. Sherman MR, Saifer MG, Perez-Ruiz F. PEG-uricase in the management of treatment-resistant gout and hyperuricemia. Adv Drug Delivery Rev 2008;60:59-68.

70. Tan QY, Wang N, Yang H, Zhang LK, Liu S, Chen L, et al. Characterization, stabilization and activity of uricase loaded in lipid vesicles. Int J Pharm 2010;384:165-72.

71. Zhao Y, Zhao L, Yang G, Tao J, Bu Y, Liao F. Characterization of a uricase from Bacillus fastidious A. T. C. C. 26904 and its application to serum uric acid assay by a patented kinetic uricase method. Biotechnol Appl Biochem 2006;45:75-80.

72. Budai M, Chapela P, Gróf P, Zimmer A, Wales ME, Wild JR, et al. Physicochemical characterization of stealth liposomes encapsulating an organophosphate-hydrolysing enzyme. J Liposome Res 2009;19:163-8.

73. Zhou Y, Zhang M, He D. Uricase alkaline enzymosomes with enhanced stabilities and anti-hyperuricemia effects induced by favourable microenvironmental changes. Sci Rep 2016;7:1-14.

74. Walde P, Ichikawa S. Enzymes inside lipid vesicles: preparation, reactivity and applications. Biomol Eng 2001;18:143-77.

75. Tan QY, Wang N, Yang H. Preparation and characterization of lipid vesicles containing uricase. Drug Delivery 2010;17:28-37.

76. Szczurek P, Mosiichuk N, Woliński J. Oral uricase eliminates blood uric acid in the hyperuricemic pig model. PLoS ONE 2017;12:179-95.

77. Grassi D, Ferri L, Desideri G. Chronic hyperuricemia, uric acid deposit and cardiovascular risk. Curr Pharm Des 2013;19:2432-8.

78. Xiong H, Zhou Y, Zhou Q. Nanosomal micro assemblies for highly efficient and safe delivery of therapeutic enzymes. ACS Appl Mater Interfaces 2015;7:20255-63.

79. Liu X, Zhang Z, Zhang Y. Artificial metalloenzyme-based enzyme replacement therapy for the treatment of hyperuricemia. Adv Funct Mater 2016;26:7921-8.

80. Marcus AJ, Broekman MJ, Drosopoulos JH. Inhibition of platelet recruitment by endothelial cell CD39/ecto-ADPase: significance for occlusive vascular diseases. Ital Heart J 2001;2:824-30.

81. Chaikof EL, Haller CA, Cui1 W, Wen J, Robson SC. CD39 enzymosomes inhibit platelet activation in vitro and in vivo. J Surg Res 2006;130:234-5.

82. Nielsen UB, Kirpotin DB, Pickering EM, Drummond DC, Marks JD. A novel assay for monitoring internalization of nanocarrier coupled antibodies. BMC Immunol 2006;7:1-15.

83. Jing Y, Trefna HD, Persson M, Svedham S. Heat-activated liposome targeting to streptavidin-coated surfaces. Biochim Biophys Acta 2015;1848:1417-23.
84. Howard M, Zern BJ, Anselmo AC. Vascular targeting of nanocarriers: perplexing aspects of the seemingly straightforward paradigm. ACS Nano 2014;8:4100-32.

85. Powers AD, Palecek SP. Protein analytical for diagnosing, monitoring, choosing treatment for cancer patients. J Healthcare Eng 2012;3:503-34.

86. Su Y, Xie Z, Kim GB, Dong C, Yang J. Design strategies and applications of circulating cell-mediated drug delivery systems. ACS Biomater Sci Eng 2015;1:201-17.

87. Dowhan W. Understanding phospholipid function: why are there so many lipids? J Biol Chem 2017;292:10755-66.

88. Collins MD, Gordon S. Giant liposome preparation for imaging and patch-clamp electrophysiology. J Visualized Exp 2013;50:1-9.

89. Kube S, Hersch N, Naumovska E. Fusogenic liposomes as nanocarriers for the delivery of intracellular proteins. Lagmuir 2017;33:1051-9.

90. Smith E, Collins I. Photoaffinity labeling in target-and bindingsite identification. Future Med Chem 2015;7:159-83.

91. Fogen D, Wu SC, Ng KKS, Wong SL. Engineering streptavidin and streptavidin binding peptide with infinite binding affinity and reversible binding capability: purification of a tagged recombinant protein to high purity via affinity-driven thiol coupling. PLoS One 2015;10:137-9.

92. Barbet J, Machy P, Leserman LD. Monoclonal antibody covalently coupled to liposomes: specific targeting to cells. J Supramol Struct Cell Biochem 1981;243:16-23.

93. Picktel E, Niemirowicz K, Watek M. Recent insights in nanotechnology-based drugs and formulations designed for effective anti-cancer therapy. J Nanobiotechnol 2016;14:1-23.

94. Zhao C, Busch DJ, Vershel CP, Stachowiak JC. Multi-functional transmembrane protein ligands for cell-specific targeting of plasma membrane-derived vesicles. SMALL 2016;12:3387-48.

95. Jain S, Gautam V, Naseem S. Acute-phase proteins: as a diagnostic tool. J Pharm Bioall Sci 2011;3:118-27.

96. Cherian AK, Rana AC, Jain SK. Self-assembled carbohydratestabilized ceramic nanoparticles for the parenteral delivery of insulin. Drug Dev Ind Pharm 2000;26:459-63.

97. Rahmanian N, Eskandani M, Barar J, Omidi. Recent trends in targeted therapy of cancer using grapheme oxide-modified multifunctional nanomedicines. J Drug Target 2017;25:202-15.

98. Korting SM, Mehnert W, Korting HC. Lipid nanoparticles for improved topical application of drugs for skin diseases. Adv Drug Delivery Rev 2007;59:427-43.

99. Sutariya V, Patel P. Aquasome: a novel carrier for drug delivery. Int J Pharm Sci Res 2012;3:688-91.

100. Nair SC, Nair AS, Vidhya KM, Saranya TR, Sreelakshmy KR. Emulsomes: a novel liposomal formulation for sustained drug delivery. Int Res J Pharm Appl Sci 2013;3:192-6. 